\title{
RETRACTED ARTICLE: Total facial nerve decompression in severe idiopathic recurrent facial palsy
}

\author{
Kai Ai $\cdot$ Mai Sun $\cdot$ Yonggang Liu $\cdot$ Bowen Guo $\cdot$ \\ Menjiang Tu $\cdot$ Jiapeng Zhao $\cdot$ Bing Xiao
}

Received: 26 July 2014/ Accepted: 8 October 2014/Published online: 24 October 2014

(c) Springer-Verlag Berlin Heidelberg 2015

The Editor in Chief and the Publisher have decided to retract this article. Upon investigation carried out according to the Committee on Publication Ethics guidelines, it has been found that the authors have duplicated substantial parts from the following article: 'Subtotal facial nerve decompression in preventing further recurrence and promoting facial nerve recovery of severe idiopathic recurrent facial palsy', by Shu-hui Wu, Xiang Chen, Jie Wang, Hua Liu, Xiao-zhong Qian, Xin-liang Pan, DOI:10.1007/ s00405-014-2991-9.

K. Ai $\cdot$ M. Tu

Department of Urology, The Second Xiangya Hospital of

Central South University, Changsha, China

M. Sun

Department of Otolaryngology, Xiangya School of Medicine, Central South University, Changsha, China

Y. Liu

Department of Sports Medicine, Hengyang Normal College,

Hengyang, China

B. Guo

Xiangya School of Medicine, Central South University,

Changsha, China

J. Zhao

Department of Neurosurgery, Xiangya School of Medicine,

Central South University, Changsha, China

B. Xiao $(\square)$

Department of Otolaryngology, The Second Xiangya Hospital of Central South University, Changsha 410011, Hunan, People's

Republic of China

e-mail: xiaobing201409@163.com 